\title{
Study of Manufacturing Processes for Liquid Rocket Turbopump Impellers: Test and Analysis
}

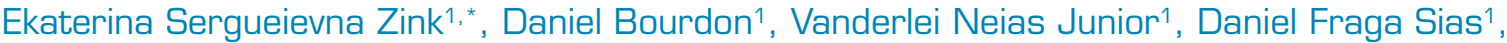 \\ Wolfgang Kitsche ${ }^{2}$, Bernd Wagner
}

Zink E (D) https://orcid.org/0000-0001-6770-4174

Bourdon D (D) https: / /orcid.org/0000-0001-6232-7779

Neias Junior V iD https: / /orcid.org/0000-0002-2132-7753

Fraga Sias D iD https://orcid.org/0000-0003-3152-028X

Kitsche W (D) https://orcid.org/0000-0003-2918-8548

Wagner B (iD https://orcid.org/0000-0003-3431-5898

\section{How to cite}

Zink ES; Bourdon D; Neias Junior V; Fraga Sias D; Kitsche W; Wagner B (2020) Study of Manufacturing Processes for Liquid Rocket Turbopump Impellers: Test and Analysis J Aerosp Tecnol Manag, 12: eO920. https://10.5028/jatm.v12.1099

\begin{abstract}
In the context of the cooperative development of the L75 Liquid Rocket Engine (with 75 kN of thrust) and in the frame of a global enlargement of competence in the field of turbomachines for liquid rocket propulsion systems, Institute of Space Propulsion of the German Aerospace Center (DLR; in German, Deutsches Zentrum für Luft- und Raumfahrt), in Lampoldshausen, and the Brazilian Institute of Aeronautics and Space (IAE; in Portuguese, Instituto de Aeronáutica e Espaço) have managed to produce its first flight ready components for pumps in liquid rocket propulsion systems. Among these components was a series of prototyped shrouded impellers for the oxidizer pumps manufactured in different materials and by different fabrication processes executed by four independent Brazilian and German workshops. Non-destructive and destructive testing methods have been applied for impellers materials validation, and for manufacturing processes verification. A special attention was given to the spin tests results and analysis, which included verification at maximum operational speed and burst testing, in order to determine the failure speed. Each tested impeller reached the required specification for the application in the L75 turbopump. Spin test logic and test results are discussed later on. Therefore, the scope of the present study is to investigate several traditional and cutting-edge processes applied for shrouded turbopump impellers manufacturing. A verification and validation of these processes will also be discussed.
\end{abstract}

KEYWORDS: Liquid rocket propulsion, L75 engine, Turbopump, Impeller, Manufacturing process.

\section{INTRODUCTION}

Liquid rocket engines are normally designed for a high combustion pressure, typically around a hundred bar, and for some types and cycles of propulsion systems far higher than this. The state-of-the-art method to provide this combustion pressure is a set of turbomachines within the propulsion system. The conditions and requirements for these turbomachines are very special compared to requirements for industrial turbopumps and turbines, since the rotational speed and the pressure raise per stage are higher than at conventional hardware. 
Thus, each pump and turbine for a rocket engine are specifically designed for their application, and off-the-shelf products cannot be used. Nevertheless, the special requirements and a very high reliability for the use in rocket engines have to be matched. Therefore, a special development for these items is necessary.

Although the design of all components of the turbopump has to be harmonized, the impeller is the key component, and special attention has to be drawn to its hydraulic design and manufacturability (Zink et al. 2017).

In the last decade, IAE (Brazil), under financial support of the Brazilian Space Agency (AEB; in Portuguese, Agência Espacial Brasileira) and in a partnership with the DLR, achieved major goals in the development project of the L75 engine. This project aims to develop, build, and qualify a biofuel liquid propellant rocket engine demonstrator (Almeida and Pagliuco 2014). The L75 engine is a liquid propellant rocket engine with a thrust of $75 \mathrm{kN}$ for an upper stage of a satellite launch vehicle. The operational concept of this engine foresees a gas generator cycle scheme, using liquid oxygen, and ethanol as propellants. The turbopump is composed of an oxidizer pump, a fuel pump, and an axial-flow turbine mounted on the same shaft. The scheme of the L75 engine turbopump is shown in the Fig. 1. The manufacturing process of the L75 turbopump LOx impeller is the issue of the present study.

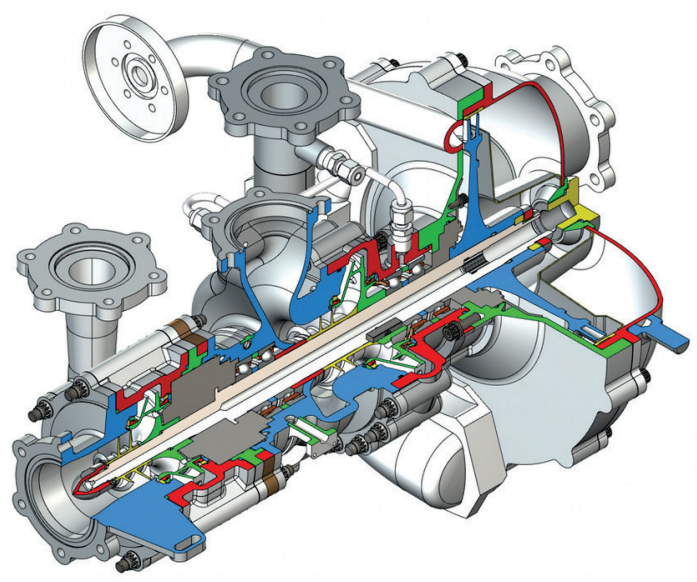

Figure 1. L75 rocket engine turbopump scheme.

Since 2013, the Institute of Space Propulsion of DLR has raised its competence to perform the development chain of pumps and turbines in liquid rocket engines. The competence includes the pre-design and the detailed design of components, flow (Computational Fluid Dynamics; CFD) and structural analysis (Finite Element Method; FEM), the manufacturing process and the examination of prototype components. For the latter, DLR also took the opportunity to gather experience in advanced manufacturing methods, such as additive layer manufacturing (ALM) and in application of advanced inspection methods such as computer tomography (CT). At the same time, the Institute of Aeronautics and Space acquired competences in turbopump development, as well as experience in the brazing process for hardware manufacturing. In the cooperation project of the Brazilian L75 rocket engine, IAE and DLR joined their efforts and expertise to investigate different shrouded impeller manufacturing processes.

\section{PROBLEM DESCRIPTION}

\section{IMPELLERS FOR TURBOPUMPS IN LIQUID ROCKET ENGINES}

The impellers of turbomachines deliver the required propellant flow and pressure to the thrust chamber of rocket engines. They are designed as radial flow components. Most of the impellers consist of a hub carrying the bladed flow channels and a 
shroud which closes the flow channels to the casing side. This design provides a well ducted flow of the fluid. Due to the high speed of the impeller, the fluid tends to evaporate and to produce cavitation at the suction side of the impeller blades (NASA 1973).

One of the most challenging engineering tasks is the attachment process of shrouds and blades, which has to meet the requirements of high rotational speed, high pressure, and cryogenic temperature.

The following chapters present an investigation of different manufacturing processes for a shrouded impeller, as it can be seen in Fig. 2, for the LOx pump application.

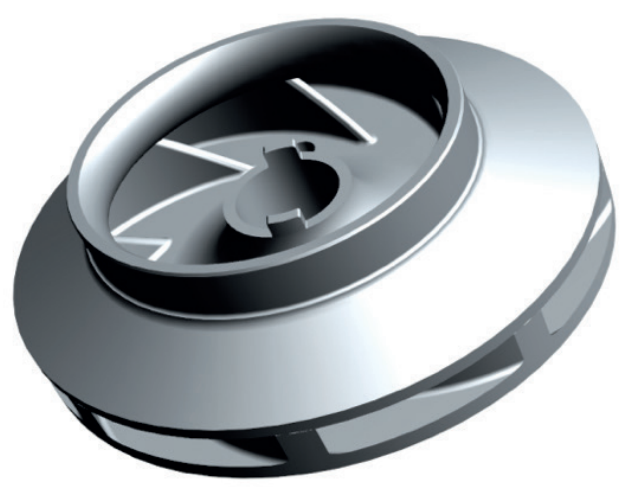

Figure 2. High speed impeller for the LOx pump of the L75 rocket engine.

\section{MATERIAL AND MANUFACTURING CHOICE CRITERIA FOR LIQUID ROCKET ENGINE TURBOPUMP IMPELLERS}

The choice of the material for a pump impeller has to follow the chemical compatibility requirements of the pump fluid, the structural requirements according to the rotor dynamic and the fluid dynamic loads, and the feasibility requirements within the fabrication process, including the required dimensional accuracy and surface quality. For fluid compatibility reasons, a liquid oxygen (LOx) impeller might not be made of aluminum or titanium. The risk of ignition and burn of these metals, in case of particle impact or rubbing in pure oxygen, is not acceptable. Inconel 625 is considered to be sufficiently ignition resistant and was selected here as the target material for oxidizer pump impellers. Even better ignition resistance can be obtained with, for example, Monel or copper alloys (NASA 1996), but they are not acceptable for structural reasons. However, other criteria, like cost and easiness of manufacturing, have also to be taken into account.

Furthermore, fuel pump impellers might feature higher speed conditions than the LOx impellers. Rather than the rotational speed, the decisive criteria and limit for the material stress is the tip speed of the impeller. Titanium is a material with very good strength and moderate material density. Therefore, titanium is often used as target material for fuel impellers. Nevertheless, for moderate requirements (concerning tip speed), high strength stainless steel might also be used for fuel impellers.

A precipitation hardening $(\mathrm{PH})$ stainless steel is a good choice for turbopump assembly (TPA) impellers, due to its availability in the market, reasonable chemical compatibility with oxygen and good mechanical properties for high speed rotating component application. Although the fuel impeller does not need to be compatible with oxygen, the same material can be selected for both impellers in order to simplify the manufacturing process.

For brazing process, however, it is important that the chosen material has the temperature of its heat treatment close to that of the brazing process. In this manner, the manufacture is simplified, and the brazing process does not impair the material properties of the impeller. This is the case for PH stainless steel, which has the solubilization temperature near to the filler metal melting temperature. Furthermore, it can be hardened after solubilization to reach high strength for fuel impeller or to reach high fatigue properties at cryogenic temperature for LOx impeller application. 
For electrical discharge machining (EDM) process, the PH stainless steel shows a good metal removal rate, and contains low carbon $(<0.07 \%-w t)$, which is desirable to reduce a material surface integrity defect known as white layer.

In addition, austenitic stainless steel has been used, during the development described here, to manufacture the L75 LOx impellers too. Although this stainless steel is not recommended for the application in the turbopump, due to its low strength, it is cost efficient to investigate and optimize a fabrication process based on this material.

The 718 nickel superalloy was used in the impellers manufactured by ALM process, due to its very high strength, good mechanical properties at cryogenic temperature, well-known behavior in high speed rotational parts application, mostly in turbopump components, and the ALM pre-developed construction parameters.

\section{MANUFACTURING PROCESSES}

There are a few types of manufacture methods for shrouded pump impellers for high speed application. The decision was made taking into account the impeller size and geometry, mainly the channel width and length. It was considered to produce the impeller integrally or the cover separately from the hub and blades. In the case of producing non-integrated impeller, it can be directly manufactured by a five-axis milling machine. The integrated impeller can have the channels made by electrical erosion machine (EEM), depending on the channels width, or can be made entirely by ALM, which has special geometry requirements that might be considered in the early design phase. In the case of producing the shroud separately, the blades can be milled from the hub in a five-axis machine, and the shroud can be joined by various methods, depending on the impeller geometry and the stress level in the region of the joint.

Typically, the parts are joined by welding, either slot welding, electro-beam welding, and laser welding, or can be joined by brazing. The welding methods are simpler, but present limitation of materials - especially those of high strength -, can require additional heat treatments, and can introduce certain level of internal stresses in the region near the joint. The brazing method, in comparison, needs special tools and furnace, but provides better dimensional stability, surface finishing, and material properties at the joint.

Casting and high pressure sintering processes were not considered for this study. Usually, they do not provide the necessary mechanical properties to the material for application in high speed rotational parts.

The main requirements that the manufacturing processes of high-speed pump impellers shall meet are dimensional tolerances, material properties and integrity, chemical and mechanical homogeneity, surface finishing, internal stress, mass balancing, cost, and lead time.

The LOx pump impeller of the L75 turbopump was produced by four different manufacturing processes as follows and summarized in Table 1.

Table 1. Overview of materials, manufacturing processes and purpose of studied impellers.

\begin{tabular}{|c|c|c|c|}
\hline Impeller & Material & Manufacturing process & Purpose \\
\hline A & PH St St & Two parts, joined by brazing & L75 turbopump \\
\hline B & PH St St & Electrical disharge machining in one part & Feasability study \\
\hline C & 321 St St & Two parts, joined by electronic welding & Feasability study \\
\hline D & Nickel alloy 718 & Selective laser melting in two parts & Lackup \\
\hline
\end{tabular}

St St $=$ stainless steel

\section{CONVENTIONAL MILLING}

The LOx pump impeller was produced by conventional methods using multi-axis computer numerical control (CNC) milling machines in different workshops. 
Figure 3 shows a computer-aided design (CAD) drawing of the hub and the blades for the milling process. The shroud was manufactured separately on a CNC lathe machine, and both parts were joined by two different processes: brazing and welding.

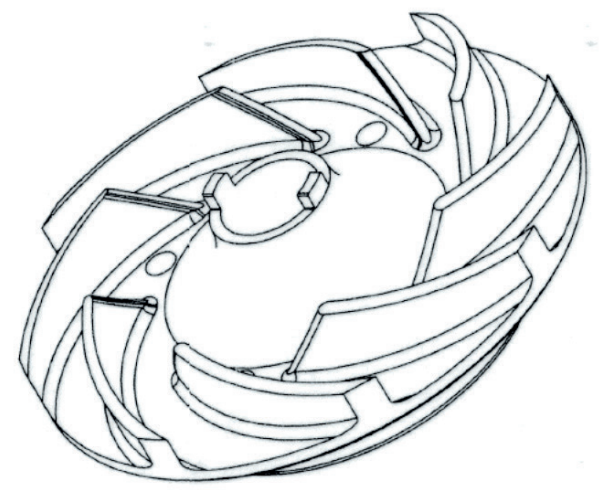

Figure 3. LOx pump impeller hub and blades.

\section{JOINING BY BRAZING}

IAE has been developing brazing processes for application in aerospace components, especially in thrust chambers of liquid rocket engines. Taking advantage of this know-how, the brazing process was used to join the shroud with the rotor of the L75 LOx pump impeller.

Brazed joints are usually strong and ductile, and can join a wide range of wall thickness. Furthermore, they do not show heat affected zone (HAZ) or dimensional distortion as the heating cycle is carried out in a vacuum furnace under controlled temperature condition.

The brazing process used in the impeller follows the operation shown in Fig. 4. The surface is prepared for the brazing process and special care is taken to remove any kind of contaminants. After cleaning, the impeller hub and shroud were assembled in the brazing device, and the brazing filler metal was applied.

The brazing cycle is developed according to the chosen filler metal and the material of the substrate (impeller). The cycle temperature was chosen to reach both the melting of the filler metal and the solubilization of the PH stainless steel at the same time. The time at melting temperature and the velocity of heating and cooling are critical parameters. Therefore, thermal gradients in the impeller during heating or cooling can cause dimensional distortion. On the other hand, slow cooling harms solubilization and can impair the material properties. In the same way, wrong duration at the filler metal melting temperature can damage the metal diffusion and harm the mechanical properties of the joint (AWS C3.3. 2002; AWS 1991).

Therefore, brazing specimens were used to find the optimum brazing cycle by many tests for the application in impellers. The impeller material was subsequently age-hardened and, then, it was machined to its final dimensions. Finally, impellers were balanced according to the standard ISO 1940-1 to a quality level required for high speed applications.

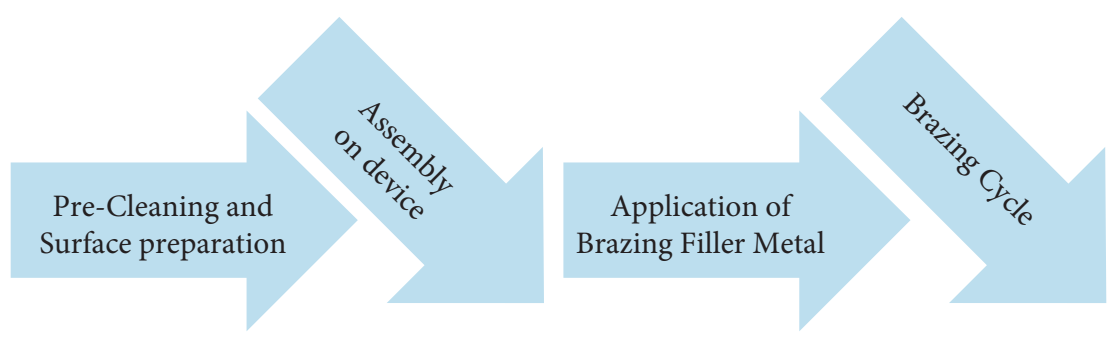

Figure 4. Brazing process. 


\section{JOINING BY WELDING}

An alternative approach was chosen by DLR to connect the hub to the shroud. Due to good experience during thrust chamber manufacturing at the Institute of Space Propulsion, electron-beam welding (EBW) was applied to connect the two parts of hub and shroud. In Murray et al. (2014), for instance, a similar procedure is proposed, but has never been applied to high speed impellers.

For further experience gain and to set up the specific parameters, geometrical test devices were manufactured and welded. The optimum parameters were chosen by observing grinding surface patterns of the welding seams. The 321 stainless steel was also chosen, because of the availability of general welding parameters and its easiness to weld.

The shroud was joined on the blade tips by CNC controlled EB-welding process through the shroud material. The thermal impact is low during the EBW process, and only the shroud was machined after welding to achieve the required surface quality.

\section{SELECTIVE LASER-MELTING, AN ADDITIVE LAYER MANUFACTURING PROCESS}

At a DLR workshop, eight impellers were printed in a M1-cusing machine by laser-concept GmbH. Six pretest impellers and two finalized ones were printed in nickel alloy. First of all, the necessity of supporting structures was confirmed for printing the impeller geometry in one part. An experimental printing was made using an impeller with stretched geometry along its axis (Fig. 5).

So far, the changed geometry is the only way to produce this impeller in one part and without supporting structures in an additive process (Fig. 6). It should be mentioned that this impeller has not the required hydraulic functionality. However, this is an experience to be considered in the predesign process of the impeller. It means that the design and manufacturing capabilities with additive processes should be integrated in the concept phase. In the present case, the supporting structures were difficult to remove from inside of the flow channels. Therefore, an alternative way was applied: the impeller was printed in two parts, which were connected finally by welding with the laser within the same machine. The duration (excluding welding) for printing a complete impeller was 36 hours. After printing, the impellers were treated by hot isostatic pressing (HIP) to improve internal integrity of the impeller material. The surface roughness was reduced by several methods, including electropolishing, grinding,
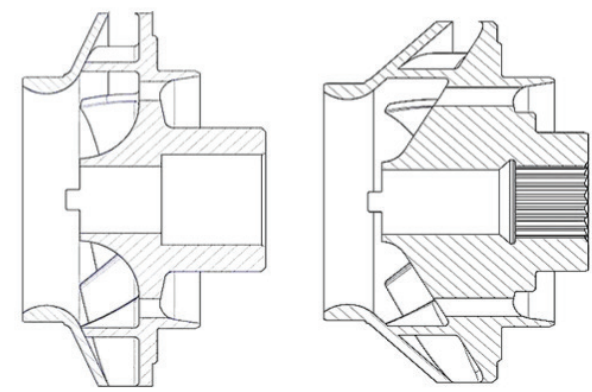

Figure 5. Original (left) and experimental impeller with stretched geometry (right).

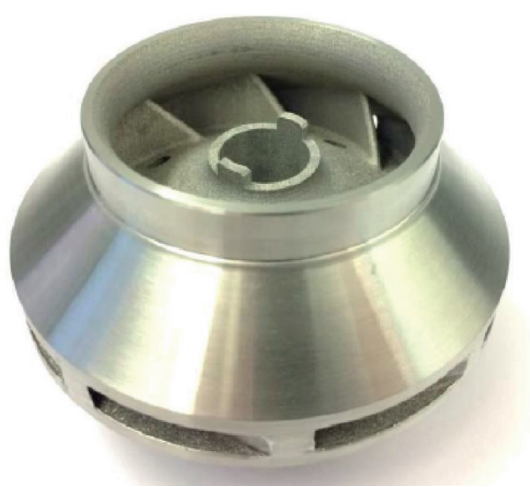

Figure 6. Printed and finalized experimental impeller, stretched and non-functional design, printed in one part. 
and sandblasting. Finally, the outer surfaces were conventionally machined to the required finishing. The coupling geometry to the shaft was added on by electric discharge eroding.

\section{EDM PROCESS}

EDM was applied for material removal, and, during this process, an electrical discharge is produced between two electrodes which are immersed in a dielectric fluid. One of the electrodes is the machining tool, the other is the raw impeller.

This process allows the manufacturing of an one-piece hardware, with complex geometry, which makes it attractive for aerospace industry, as well as for the application studied here.

The EDM process is divided into the following steps:

1. Electrodes ignition;

2. Plasma channel creation;

3. Fusion and evaporation of material on the electrodes;

4. Removal of cast material from electrode cavities.

For impeller manufacturing, five-axis electrical discharge machining was used. The electrodes have the reduced form of the blade profile in order to obtain sufficient space for the electrode move. Following a pre-established path, the electrode removes material and forms the impeller channels. To guarantee a designed thickness of the impeller parts, the electrode is divided in several segments, each one designated to manufacture one section of the impeller cavity. Due to the complexity of the impeller geometry, eight different copper and graphite electrodes were used. A manual polishing was applied to obtain the required surface finish. Again, the impellers were balanced according to the standard ISO 1940-1.

\section{EXAMINATION PROCESSES}

Altogether, six examination methods were applied to the L75 LOx impellers, in order to identify possible failures, and to evaluate the product and the fabrication process. Table 2 summarizes the different methods and their application.

Table 2. Summary of applied examination methods.

\begin{tabular}{|c|c|c|c|c|c|c|}
\hline \multirow{2}{*}{ Method } & \multicolumn{4}{|c|}{ Non-destructive analysis } & \multicolumn{2}{|c|}{ Destructive analysis } \\
\hline & CMM & LPI & Balance & X-ray & Metallography & Burst \\
\hline Impeller A & $\mathrm{x}$ & o & o & - & $\mathrm{x}$ & $\mathrm{x}$ \\
\hline B & $\mathrm{x}$ & - & o & - & - & $\mathrm{x}$ \\
\hline $\mathrm{C}$ & $\mathrm{x}$ & o & o & o & $\mathrm{x}$ & $\mathrm{x}$ \\
\hline $\mathrm{D}$ & $\mathrm{x}$ & - & o & o & - & $\mathrm{x}$ \\
\hline
\end{tabular}

$\mathrm{CMM}=$ coordinate measuring machine; $\mathrm{x}=$ performed; $\mathrm{o}=$ performed before and after spin test; $-=$ not performed .

\section{NON-DESTRUCTIVE ANALYSIS}

The manufacturing process was assisted by the coordinate measuring machine (CMM) technique, in order to inspect the main blade geometric parameters, such as angular position of the blade channels, and thickness and curvatures of the blades. The surface roughness and outer dimensions after joining were also measured.

Liquid penetrant inspection (LPI) is often used to detect discontinuities extending to the surfaces of brazed joints. That makes it possible to find cracks and porosity in the brazed surface if the process was not effective. 
After brazing, the impellers passed standardized inspections (AWS C3.3. 2002) including LPI. It does not show any type of porosity or cracks. The same method was applied after the spin tests for brazed and selected welded impellers, and no indication for anomalies was found.

$\mathrm{X}$-ray techniques were also used to inspect the impellers. X-ray images were recorded for each welded impeller at the eight blades joints. They were manually inspected for defects, but only marks typically for the EBW process were found. The computer tomography technique was also applied, for two reasons: first, to verify the integrity of the ALM impellers; second, to survey the internal blade geometry as it cannot be accessed by CMM technique. The computer tomography (CT) resulting 3D model showed some artifacts, and the dimension could not be proofed in the requested precision. Further work will improve this helpful technique.

\section{DESTRUCTIVE ANALYSIS}

The impellers were subjected to destructive methods to evaluate and verify the brazing process. Metallographic examination is used to observe the diffusion level of the filler metal through the substrate, which provides evidences of certain properties based on procedure qualification (AWS C3.3. 2002). Four segments were cut from the impeller near the brazed joints, and metallographic analysis was performed, as demonstrated in the Fig. 7.

Figure 8 depicts metallographic inspection of the brazed specimens. Here, it can be observed that there is a diffusion of elements between filler metal and substrate. It shows the presence of a nickel reach solid solution (clear region) with a fine intermetallic phase dispersed in the joint region, which demonstrates a significant interaction between the filler alloy and the original material, as well as the establishment of a stable microstructure. Hence the joint is considered with acceptable quality (AWS 1991).
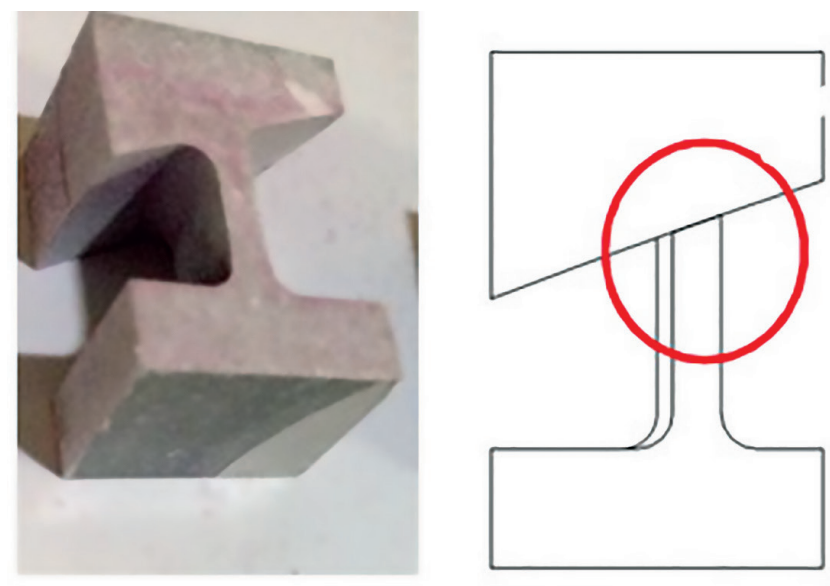

Figure 7. Segment ready for surface preparation and inspection.
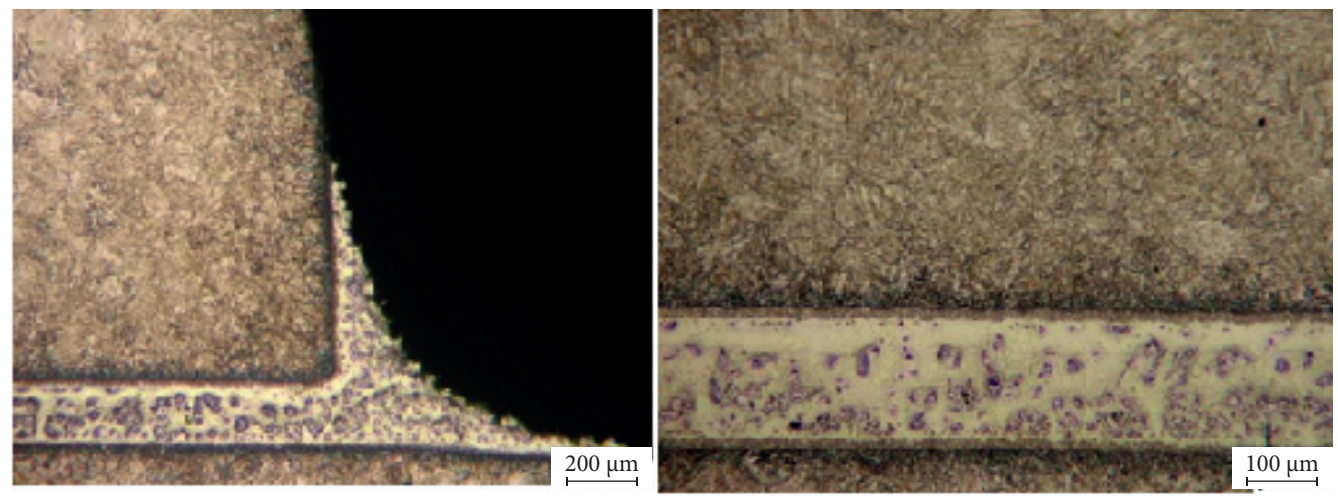

Figure 8. Metallographic inspection of brazing joint. 


\section{SPIN TESTS}

The spin test is a dynamic examination frequently used to evaluate high speed rotating parts, in order to verify its mechanical integrity during service, which is subjected to high centrifugal forces. Generally, the part is spun to its maximum operational speed under vacuum (spin test) or is gradually accelerated until it bursts (burst test), in order to determine the maximum speed it can support. Frequently, the radial deformation is measured to define the effects of speed on it. These tests can alternatively be performed at the temperature of operation and realistic conditions of vibration.

As a part of the manufacturing validation process, spin tests were planned. A German company specialized on high precision balancing and spin tests was chosen to perform this task. Two of the available spin tests machines were used, one that applies up to 60,000 revolutions per minute (rpm); and another that applies up to $120.000 \mathrm{rpm}$. Both operate the test specimen in near vacuum conditions ( $<1 \mathrm{mbar}$ ) and at ambient temperature. Temperature, shaft vibration and the rotational speed were monitored during tests.

\section{STRUCTURAL ANALYSIS}

In order to predict impeller radial deformation during spin test, a structural analysis using FEM was performed. The impeller geometry was taken from a CAD model to generate the $5 \times 10^{5}$ elements mesh using ANSYS software. The mesh was composed by solid quadratic hexahedral elements. The boundary conditions reproduced the spin test operation until $120 \%$ of nominal speed under vacuum. A fixed boundary was used on the hub rear face to reproduce the impeller clamping device. A radial load was applied to reproduce the centrifugal force. It was considered that the cover and blades are perfectly joined, and the material is homogeneous $\mathrm{PH}$ stainless steel. Initially, the modal analysis indicates that there are no critical speeds in the range of the spin test that could lead to a harmful vibration. The structural analysis indicates that the impeller will deform radially less than $25 \mu \mathrm{m}$ at the most in the shroud region, and less than $15 \mu \mathrm{m}$ in the seals surfaces. Figure 9 shows the impeller radial deformations from FEM structural analysis.

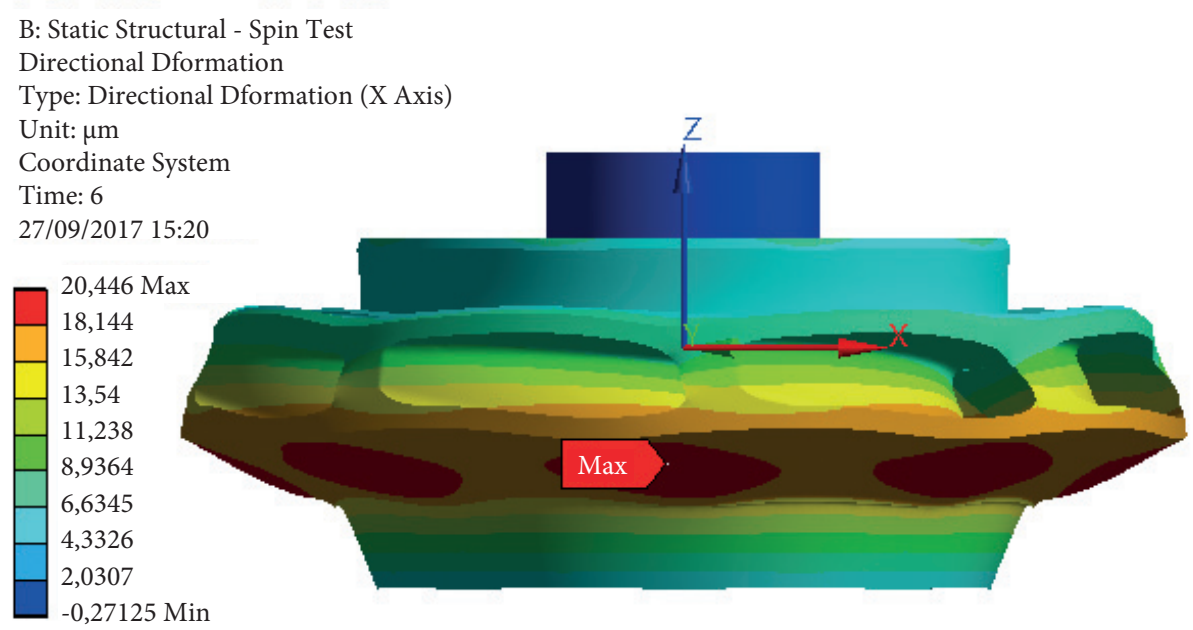

Figure 9. Impeller radial deformation induced by spin test operation condition.

\section{MEASUREMENT OF UNBALANCE}

Two different approaches were applied to deal with the balancing of the parts. Impellers A and B were balanced as part of their manufacturing process and the turbopump requirements. The unbalance of the impellers $\mathrm{C}$ and $\mathrm{D}$ was measured after the manufacturing, in order to get information about the precision, especially of the ALM process, and compare it with conventional processes.

Independently from that, all impellers pass a high precision static unbalance measurement before and after the spin test. In this way, change of mass distribution due to plastic deformation during test could be detected. However, changes of the true unbalance 
and position of the balance were an order of magnitude smaller than the re-clamping and measurement uncertainty. Only for the $\mathrm{D}$ impellers manufactured by ALM, a change of more than one gram $/ \mathrm{mm}$ was found. For the other impeller, the effect was negligible.

In order to minimize vibration and, therefore, increase the precision of the deformation measurements, the entire spin test rotor was balanced with screws, as it is shown in Fig. 10. Thus, the impellers were not modified for the tests and the changes in unbalance can be tracked.

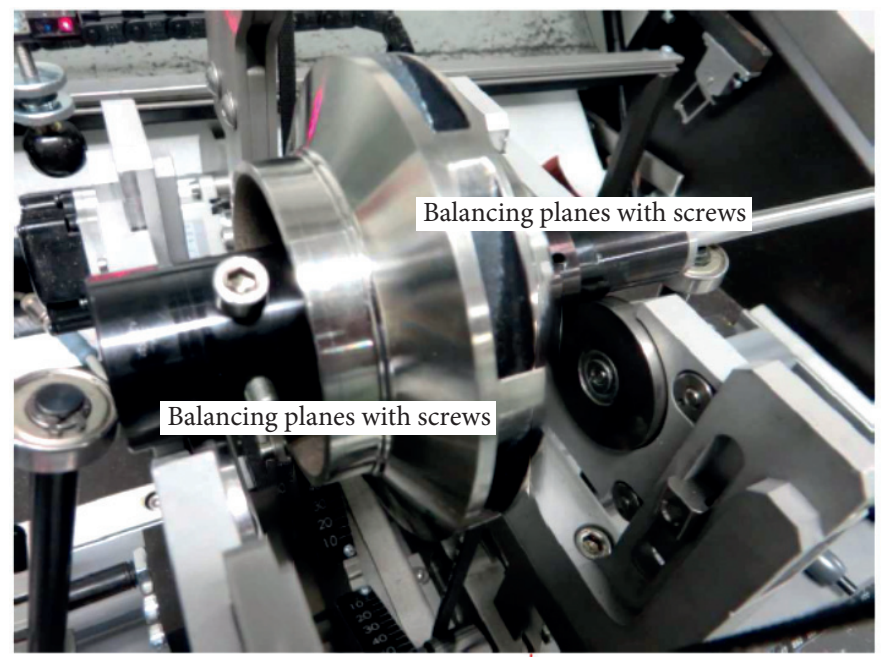

Figure 10. Impeller with adaptor on the balancing scale.

\section{DEFORMATION MEASUREMENTS}

In a first step, the spin test setup was used to measure the deformation of the impellers at particular positions. For impellers A and B, the measurement was on the impeller seal surfaces on hub and shroud side (Fig. 11).

In order to measure at the position of the highest expected deformation and to gain data for validation of FEM simulations, the sensor position was changed to the impeller outlet for impeller C and D (Fig. 12).

Capacitive high-speed displacement sensors were used during the tests. They were positioned under $180^{\circ}$ over the selected impeller surfaces. The expansion could be measured consequentially from the two positions while speeding up the rotor. A reference measurement at very low speed was chosen to calculate the expansion difference related to this reference.

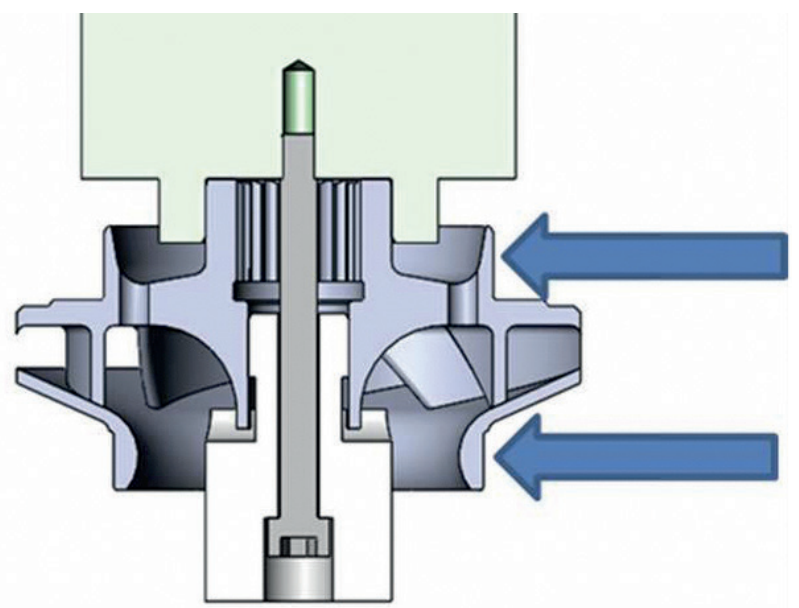

Figure 11. Measurement positions for impellers $A$ and $B$ on seal surfaces. 


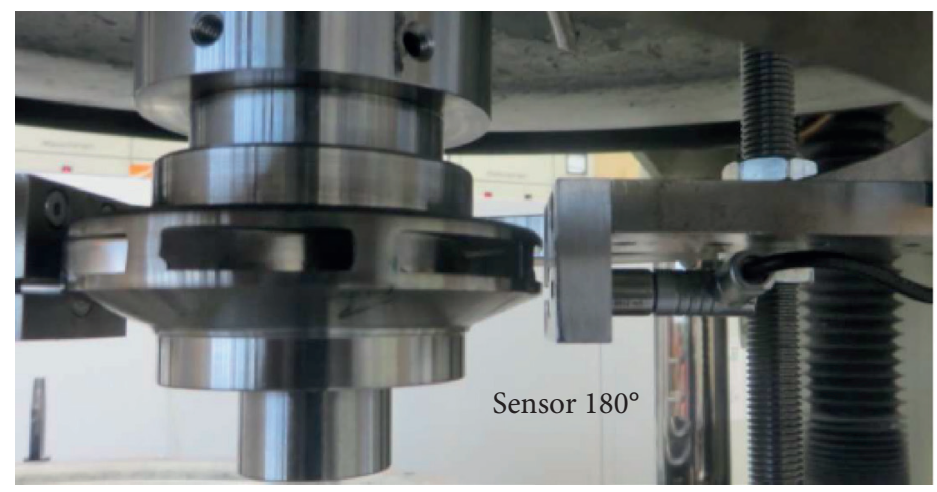

Figure 12. Sensor position for impellers $C$ and $D$.

In Fig. 13, the measured normalized deformation $\left(\delta / \delta_{\max }\right)$ is plotted over the rotational speed. The results are shown for each material and manufacturing process. Each set of results is normalized by its maximum measured deformation. At the different rotational speed steps, several measurements were performed and data was recorded. Only the first results of each speed step are used in the figure. Long operational times at $115 \%$ of speed lead to heating and, therefore, unwanted thermal expansion of the impellers. The data points are averages from 16 measurement points in circumferential direction. Maximum and minimum of these points are indicated by the error bars.

All curves show a similar parabolic shape. Due to the normalization, the differences in material and sensor position are compensated. Impellers A and B have larger deformation at 67 and $83 \%$ of nominal speed. This behavior might be explained by the occurrence of higher shaft vibrations in the spin test machine at these speed steps.

Three of type $\mathrm{C}$ and two of type $\mathrm{D}$ impellers have also been tested, but only one curve is shown for clarity in each case, because excellent agreement between the different tests could be found for average expansion over rotational speed.

The expansion measurement for those impellers has been performed at their outlet, as it can be seen in Fig. 12. At this position, the data acquisition of the capacitive sensor was influenced by the changing geometry. Thus, the sensor spot level at impeller

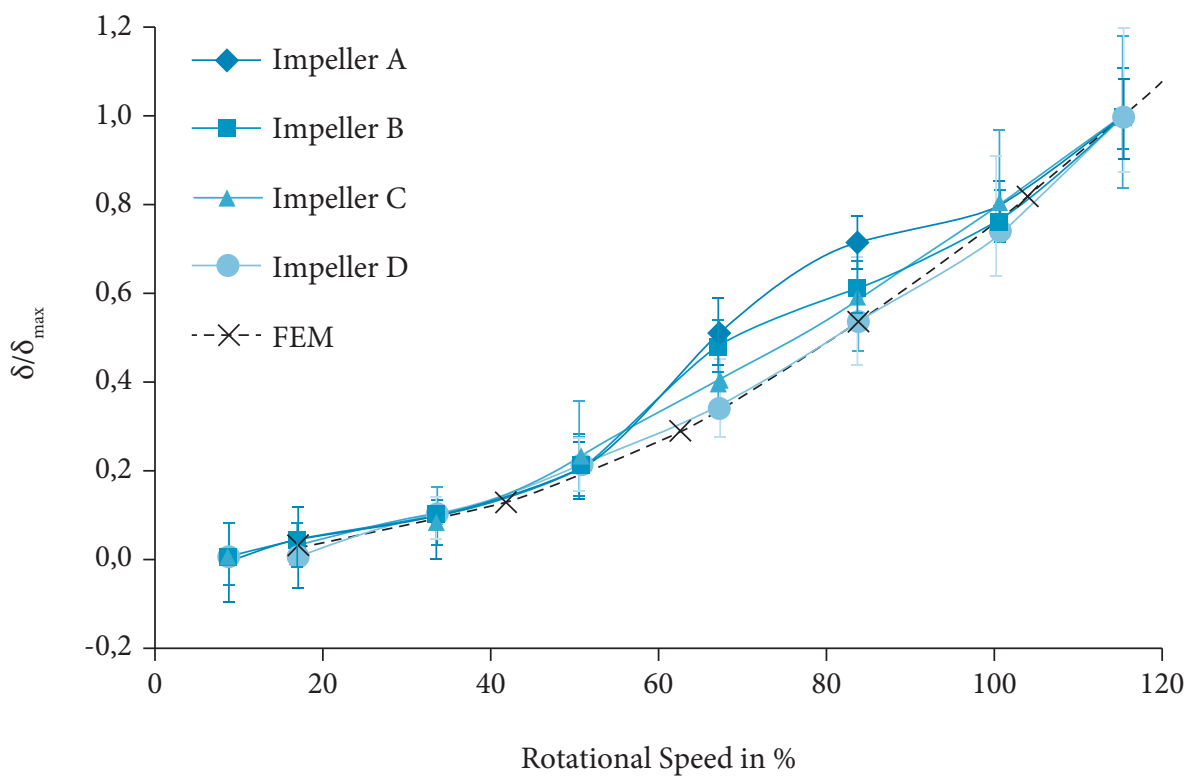

Figure 13. Normalized deformation over speed for four impellers during spin tests compared to FEM results. 
bridges (blade outlet) and over free plate is different. The averaging diminishes this effect, but it leads to larger error bars. At higher rotational speed, the different expansion values depending on the geometry are overlaying this effect.

In Fig. 14, the circumferential distribution of expansion is plotted for the same cases as shown previously. The data is normalized by each average value. Only nominal rotational speed (100\%) is presented for clearness. For the values measured at the impeller exit, the aforementioned effect is obvious. Impeller $\mathrm{C}$ shows more obvious the eight blades than impeller D case. For impellers $\mathrm{A}$ and $\mathrm{B}$, with the measurement position at the hub seal, the influence of the blades can be recognized, but it is smaller. In this case, it results only from deformation.

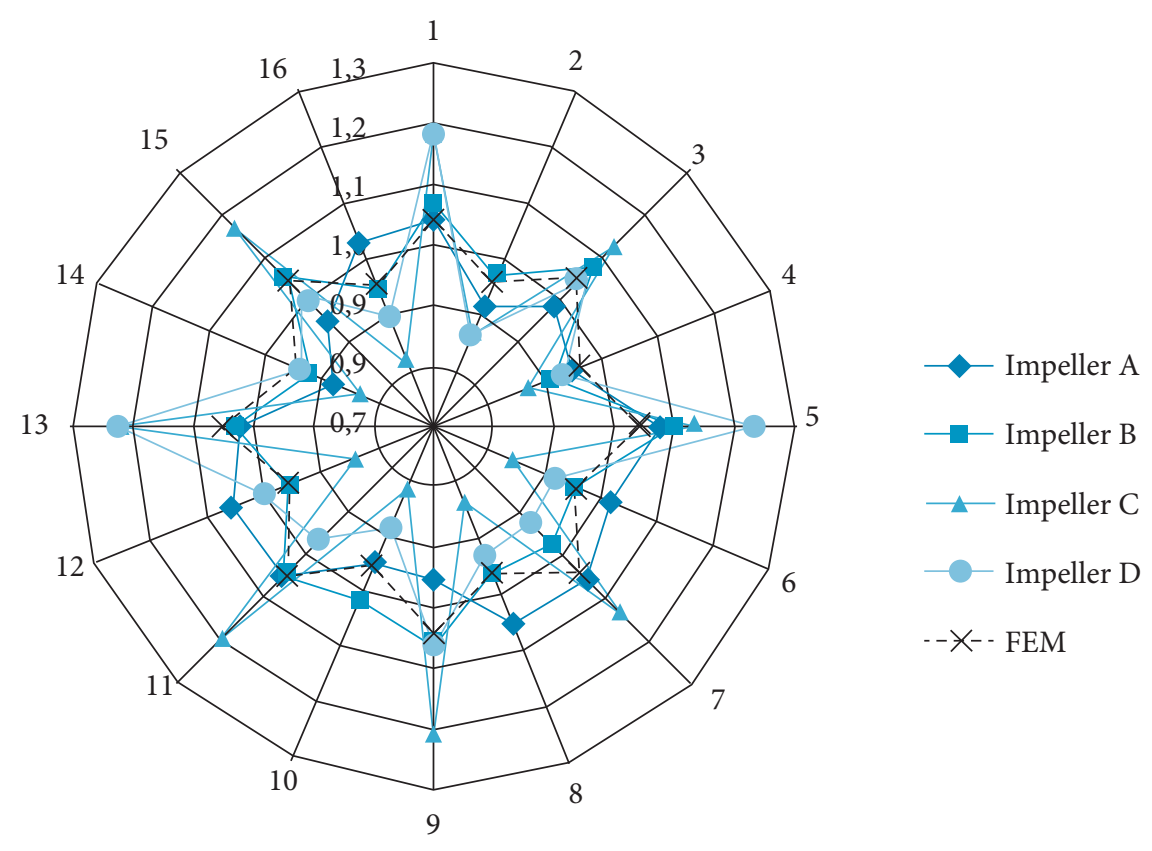

Figure 14. Normalized expansion values at circumferential directions for nominal rotational speed (100\%).

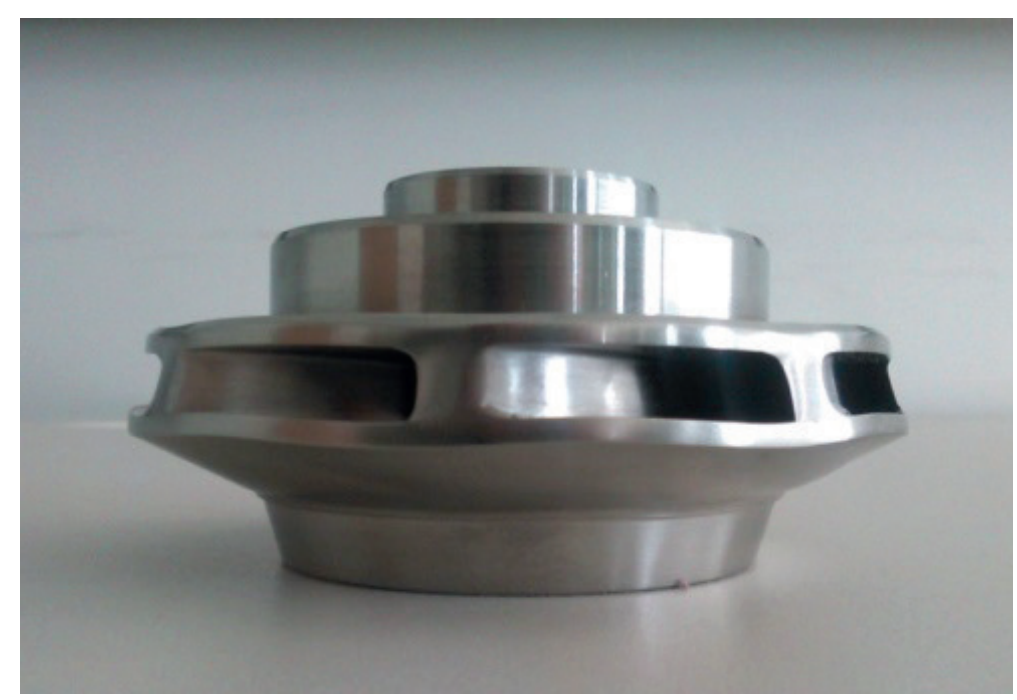

Figure 15. Strong plastic deformation of a stainless steel impeller. 


\section{BURST TESTS}

One of each set of impellers was tested towards the burst speed. Although, only one impeller desintigrated during tests, spin tests are a desctructive examination process. The results are summarised in Table 3.

Table 3. Summary of LOx impeller burst tests.

\begin{tabular}{|c|c|c|}
\hline Name & Max. speed [\%] & Comments \\
\hline Impeller A & 250 & Neither indication for plastic deformation nor cracks \\
\hline Impeller B & 250 & Neither indication for plastic deformation nor cracks \\
\hline Impeller C & 272 & Strong plastic deformation, stop due to high vibration \\
\hline Impeller D & 411 & Burst of impeller \\
\hline
\end{tabular}

Prior to the test, a structural analysis was performed and an empirical form-factor method (Lüdtke 2004) was used to calculate the expected burst speed. A lower burst speed was expected for impellers A and B, made out of PH stainless steel, and they reached the limits of the spin test machine without showing either indication of plastic deformation or cracks. Tests were not repeated on the more performant machine for those impellers.

The structural analysis indicated an early plastic deformation for impeller C, which is typical for the 321 stainless steel material without thermal treatment. During the burst test, the results revealed first plastic deformation initiating around 230 to $242 \%$ of nominal speed. The burst test was aborted at $272 \%$, after strong plastic deformations led to vibrations exceeding the maximum allowed value.

In the case of impeller $\mathrm{D}$, the expected burst-speed was pre-calculated with the form-factor method to $414 \%$ of nominal speed, and the burst occurred at $411 \%$ with a complete destruction of the shroud and the blades. The rupture occurred as just above the blade roots at the hub.

\section{CONCLUSION}

In the context of cooperation activities of the Brazilian L75 liquid rocket engine development, IAE and DLR succeeded to investigate several traditional and cutting-edge processes applied for turbopump impellers manufacturing. To verify and validate these processes, all rotors were submitted to non-destructive and destructive tests and analysis. The results obtained during verification are considered to acceptably fulfill the established design requirements. All impellers withstood a nominal turbopump rotational speed without any failure or deformation. The applied burst tests proved that the adopted manufacturing processes provide a good resistance and can support at least two times the nominal rotational speed before impeller disintegration.

Therefore, the presented manufacturing methods can be implemented, on one hand, for the L75 turbopump development in order to produce fuel and oxidizer pumps, and on the other hand, they might be applied in general for liquid rocket engine turbopumps. However, in case of mass production, additional studies should be done. For example, such criteria as workshop readiness, time and related costs shall also be considered.

\section{ACKNOWLEDGEMENTS}

The work of the different workshops at DLR SHT Göttingen, DLR Lampoldshausen and Brazilian suppliers is gratefully acknowledged. The contribution of Dietmar Maier (DLR) to this work is appreciated.

The authors wish to thank AEB/IAE for supporting all activities performed in the frame of the L75 project. 


\section{AUTHORS' CONTRIBUTION}

All the authors contributed equally to this paper.

\section{REFERENCES}

Almeida D, Pagliuco CMM (2014) Development status of L75: A Brazilian liquid propellant rocket engine. J Aerosp Technol Manag 6(4). doi: http://dx.doi.org/10.5028/jatm.v6i4.386

AWS [American Welding Society] (1991) Brazing handbook. 5th ed. Miami: AWS.

Lüdtke KH (2004) Process centrifugal compressors. Berlin: Springer-Verlag.

Murray W, Steiner M, Neal J, Hunt S (2014 July) Design and analysis of a high speed, high pressure peroxide/RP-1 turbopump. Paper presented 50th AIAA/ASME/SAE/ASEE Joint Propulsion Conference, AIAA 2014-3499; Cleveland, USA. doi: https://doi. org/10.2514/6.2014-3499

NASA [National Aeronautics and Space Administration] (1973) Liquid rocket engine centrifugal flow turbopumps. NASA SP 8109.

NASA [National Aeronautics and Space Administration] (1996) Safety standard for oxygen and oxygen systems: Guidelines for oxygen system design, materials selection, operations, storage, and transportation. Office of Safety and Mission Assurance. NASA NSS 1740.15.

Nowacki J, Swider P (2003) Producibility of brazed high-dimension centrifugal compressor impellers. J Mater Process Technol 133:174180. doi: https://doi.org/10.1016/S0924-0136(02)00228-5

Strain WS (2008) Design of an oxygen turbopump for a dual expander cycle rocket engine (MS thesis). Ohio: Air Force Institute of Thechnology.

Zink ES, Bourdon D, Almeida DS, Pagliuco CMM, Wagner B, Kitsche W, Langel G (2017) Biofuel turbopump development strategy: L75 Liquid oxygen and ethanol engine case. Paper presented 53rd AIAA/ASME/SAE/ASEE Joint Propulsion Conference, AIAA 2017-4929; Atlanta, USA. doi: https://doi.org/10.2514/6.2017-4929 